Revista Brasileira de

Engenharia Agrícola e Ambiental

v.16, n.6, p.675-683, 2012

Campina Grande, PB, UAEA/UFCG - http://www.agriambi.com.br

agriambi

Protocolo 177.11 - 22/08/2011 • Aprovado em 28/03/2012

\title{
Cinética del secado de tomillo
}

\author{
Ronicely P. da Rocha ${ }^{1}$, Evandro de C. Melo ${ }^{2}$, José B. Corbín ${ }^{3}$, \\ Pedro A. Berbert ${ }^{4}$, Sérgio M. L. Donzeles ${ }^{5}$ \& Jon A. Tabar ${ }^{6}$
}

\begin{abstract}
RESUMEN
Los objetivos del trabajo fueran estudiar la cinética del secado de tomillo con posterior ajuste de diferentes modelos matemáticos a los datos experimentales y determinar los valores de la difusividad efectiva y energía de activación. Para el desarrollo del experimento de secado fueran utilizadas las temperaturas 30, 40, 50, 60 y $70{ }^{\circ} \mathrm{C}$. El diseño experimental fue de bloques completamente aleatorizados, con tres repeticiones. Para el ajuste de los modelos matemáticos a los datos experimentales fue realizado el análisis de regresión no lineal, por el método Simplex-Quasi-Newton. Los resultados mostraron que el modelo matemático de Page obtuve un mejor ajuste a los datos experimentales, los valores de los coeficientes de difusividad efectiva variaron entre 3,69 × $10^{-12}$ e 1,19 × $10^{-10} \mathrm{~m}^{2} \mathrm{~s}^{-1}$ y el valor de la energía de activación fue de $77,16 \mathrm{~kJ} \mathrm{~mol}^{-1}$.
\end{abstract}

Palabras-clave: secado, modelos matemáticos, Thymus vulgaris L.

\section{Drying kinetics of thyme}

\begin{abstract}
This work aimed to study the drying kinetics of thyme and subsequent adjustment of different mathematical models to the experimental data and to determine the effective diffusivity and the activation energy. In order to conduct the experiment the temperatures of $30,40,50,60,70{ }^{\circ} \mathrm{C}$ were used. The experimental design employed was in randomized blocks with three repetitions. For the adjustment of the mathematical models to the experimental data non-linear regression was performed using SimplexQuasi-Newton method. The results showed that the Page model was the one that promoted the best fit of the experimental data to describe the drying kinetics of the thyme leaves. The values of effective diffusivity coefficients ranged between $3.69 \times 10^{-12}$ and $1.19 \times 10^{-10} \mathrm{~m}^{2} \mathrm{~s}^{-1}$ and the value of activation was $77.16 \mathrm{~kJ} \mathrm{~mol}^{-1}$.
\end{abstract}

Key words: drying, mathematical model, Thymus vulgaris L.

\footnotetext{
Pós-doutorando, Departamento de Engenharia Agrícola, Universidade Federal de Viçosa. Brasil. Email: ronicely.rocha@ufv.br ${ }^{2}$ Departamento de Engenharia Agrícola, Universidade Federal de Viçosa., Brasil. Email: evandro@ufv.br

${ }^{3}$ Departamento de Tecnologia de Alimentos, Universidade de Valencia, Espanha. Email: jbon@tal.upv.es

${ }^{4}$ Departamento de Engenharia Agrícola, Universidade Estadual Norte Fluminense, Brasil. Email: pbebert@uenf.br

${ }^{5}$ EPAMIG, Brasil. Email: slopes@ufv.br

${ }^{6}$ Graduando, Departamento de Engenharia Agrícola, Universidade Publica de Navarra, Espanha. Email: aspurz@hotmail.com
} 


\section{INTRODUCIÓN}

El tomillo (Thymus vulgaris L.) es una planta medicinal y aromática, perteneciente a la familia Lamiaceae, originaria de Europa y cultivada en el sur y sureste de Brasil. La familia Lamiaceae comprende 150 géneros, con cerca de 2800 especies distribuidas por todo el mundo, siendo el mayor centro de dispersión la región Mediterránea. Dentro de estos géneros cultivados se podrían destacar: Salvia officinalis, Ocimum basilicum, Origanum vulgaris, Origanum majorana, entre otras (Porte \& Godoy, 2001; Mewes et al., 2008).

Las hojas de tomillo son ampliamente utilizadas en la cocina como condimento. Esta planta también es utilizada en la industria de perfumes como aromatizante natural de licores. La actividad biológica del aceite del tomillo está relacionada con sus principales componentes, denominados timol y carvacrol. El timol tiene efecto astringente, expectorante, digestivo, antiespasmódico, antitusígeno, antibacteriano, antifungico y anti-helmíntico, en cuanto al carvacrol ha sido estudiado por sus efectos bactericidas. Estos efectos del aceite del tomillo fueron demostradas por diversos estudios como Daferera et al. (2000), Bagamboula et al. (2004), Novacosk \& Torres (2006), Sokoviæet et al. (2009) y Centeno et al. (2010).

En la etapa de pos cosecha merecen especial atención la etapa de secado ya que el manejo adecuado de esta puede evitar pérdidas y contribuir en la conservación del producto.

Después de la cosecha, se inicia un proceso de degradación en las plantas, debido al aumento de la actividad enzimática, que a su vez conduce a la degradación de los principios activos presentes en las mismas. Para minimizar estos efectos, las plantas medicinales deben secarse. La conservación de la calidad de los productos por el secado se basa en que tanto las enzimas como los microorganismos necesitan agua para realizar sus actividades. Con la reducción de la cantidad de agua disponible, hasta niveles seguros para almacenamiento se reducirá la actividad de agua y la velocidad de las reacciones químicas en el producto, así como el desarrollo de microorganismos. El alto contenido en agua en las células y tejidos de las plantas, en torno al 60 a $80 \%$, hacen que el secado tenga una importancia fundamental para evitar la fermentación o degradación de los principios activos (Christensen \& Kaufmann, 1974; Reis et al., 2003).

Los límites de temperatura en el secado para las diferentes plantas medicinales son diferentes para cada especie según la sensibilidad de los compuestos químicos que tenga y de las estructuras almacenadoras que tenga cada planta. Las altas temperaturas pueden llevar a la pérdida de los compuestos químicos de los aceites esenciales por volatización o degradación (Venskutonis, 1997; Lewinsohn et al., 1998).

Los modelos matemáticos son herramientas útiles para estimar el tiempo necesario en reducir la cantidad de agua del producto a diferentes condiciones de secado mejorando la eficiencia del proceso (Andrade et al., 2003). El ajuste de los datos experimentales a los modelos matemáticos es indispensable y que se haga para las plantas medicinales y aromáticas para obtener el modelo más adecuado para cada especie.
Con el objetivo de ajustar diferentes modelos matemáticos a los datos experimentales de secado, Barbosa et al. (2007) sometieron a las hojas de Lippia albaa secado con temperaturas de aire de secado de $40,50,60,70$, y $80^{\circ} \mathrm{C}$ y una velocidad de $0,29 \pm 0,03 \mathrm{~m} \mathrm{~s}^{-1}$. Los autores concluirían que los modelos de Page y Midilli et al. (2002) fueron los que representaban mejor la cinética de esta especie medicinal.

Se han estudiado diferentes tipos de mentas respecto a su secado. En el secado de Mentha crispa L., usando temperaturas de aire de 30,40 e $50^{\circ} \mathrm{C}$. Park et al. (2002) obtuvieron el mejor ajuste de los datos con el modelo de Page. Therdthai \& Zhou (2009) estudiaron el secado de Mentha cordifolia utilizando microondas al vacío y aire a 60 y $70^{\circ} \mathrm{C}$. Los autores utilizaron los modelos de Lewis, Page y Fick a los datos experimentales de secado y concluirían que el modelo de Page fue el que mejor describe esos datos. Arslan et al. (2010) utilizaron 3 métodos de secado (sol, microondas y aire a $50^{\circ} \mathrm{C}$ ) en hojas de Mentha $x$ piperita $\mathrm{L}$. Fueron estudiados doce modelos matemáticos y su resultado fue que los mejores ajustes a los datos experimentales fueron obtenidos mediante los modelos Midilli et al. (2002), Page e Page Modificado.

Por lo tanto los procesos de pos cosecha de plantas medicinales, aromáticas y condimentales, principalmente el secado, asumen un papel de extrema importancia en la cadena de producción, porque repercuten directamente en la calidad y cantidad de los principios activos que serán comercializados. Los principales objetivos de este trabajo son: obtener las curvas de secado en función de la temperatura del aire de secado y posterior ajuste de diferentes modelos matemáticos a losdatos experimentales; determinar los valores del coeficiente de difusión y de la energía de activación.

\section{MATERIAL Y MÉTODOS}

Las plantas de tomillo utilizadas para el desarrollo de este trabajo provienen de plantaciones del vivero comercial Albogaden, localizadas en Valencia, España. Las plantas eran cultivadas individualmente en vasos $\mathrm{y}$, antes de secado, los ramos eran cortados, se retiraban las hojas manualmente y almacenadas en sacos de polietileno (40 ìm) a $4 \pm 2{ }^{\circ} \mathrm{C}$ hasta el momento del secado (Figura 1).

Fueron seleccionadas aleatoriamente 3 muestras para analizarlas todavía in natura, para obtener el contenido de agua inicial. El contenido de agua inicial fue determinado de acuerdo a la metodología descrita por la AOAC (1997), utilizándose 5 g de muestra, en tres repeticiones en una estufa al vacío y una temperatura de $70 \pm 2{ }^{\circ} \mathrm{C}$, durante $24 \mathrm{~h}$. El secado fue realizado en el laboratorio de Grupo de Análisis y Simulación de Procesos Agroalimentarios del Departamento Tecnología de Alimentos de la Universidad Politécnica de Valencia, Valencia, España.

Fue utilizado un secador de flujo de aire ascendente (Figura 2). El secador disponía de un sistema de pesado automático que se registraba en un microcomputador cada $5 \mathrm{~min}$. Se registraba la masa del producto, la humedad relativa, temperatura del aire en el ambiente y en el secado y la velocidad del aire de secado. La velocidad del aire de secado fue de $1 \mathrm{~m} \mathrm{~s}^{-1}$ para todos los ensayos de secado y el secado se finalizaba 
A.

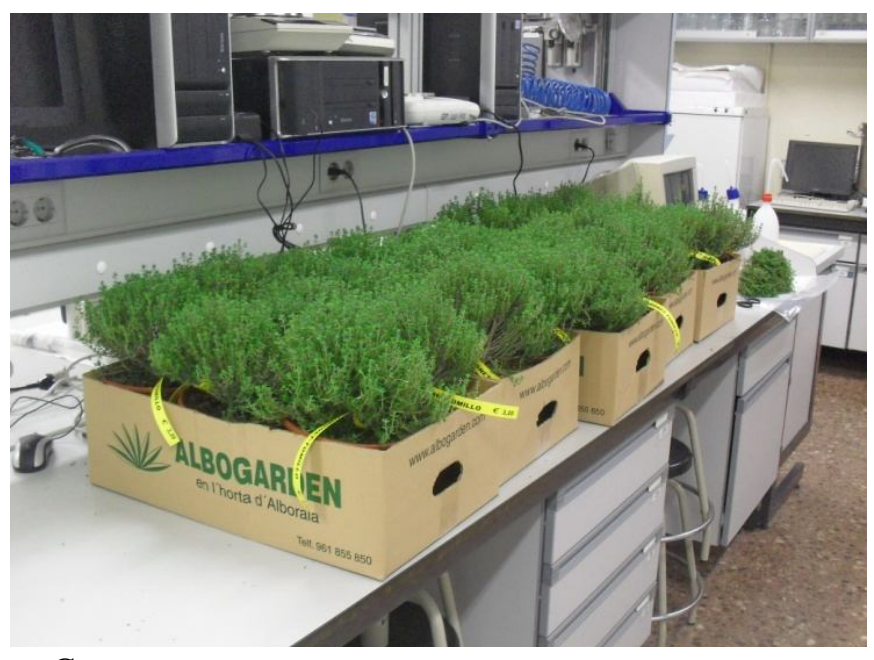

C.

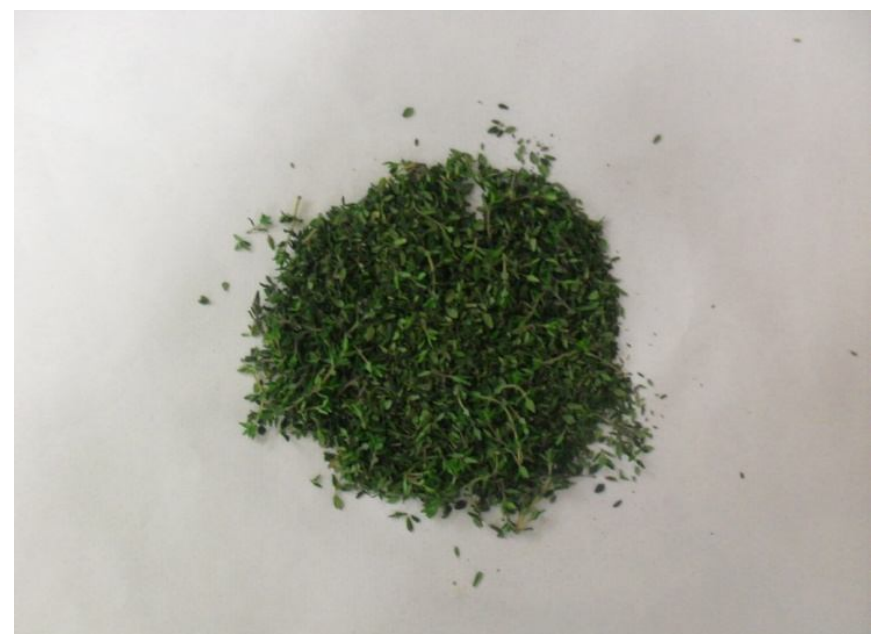

B.

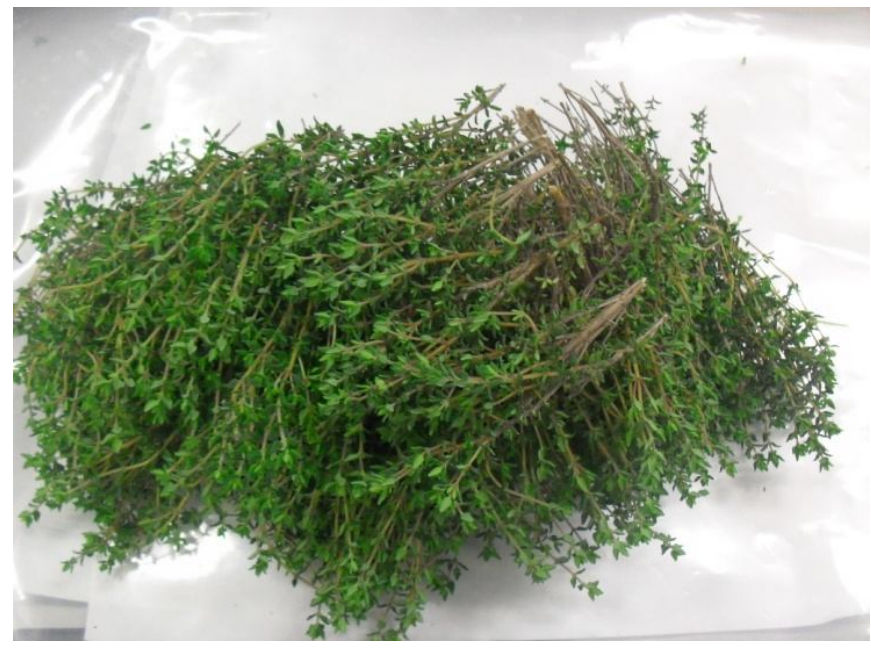

D.

Figura 1. Procedimiento con la planta del tomillo antes de iniciar los ensayos de secado. A. Planta entera de tomillo; B. Ramos con hojas de tomillo; C. Hojas; D. Hojas en sacos de plástico

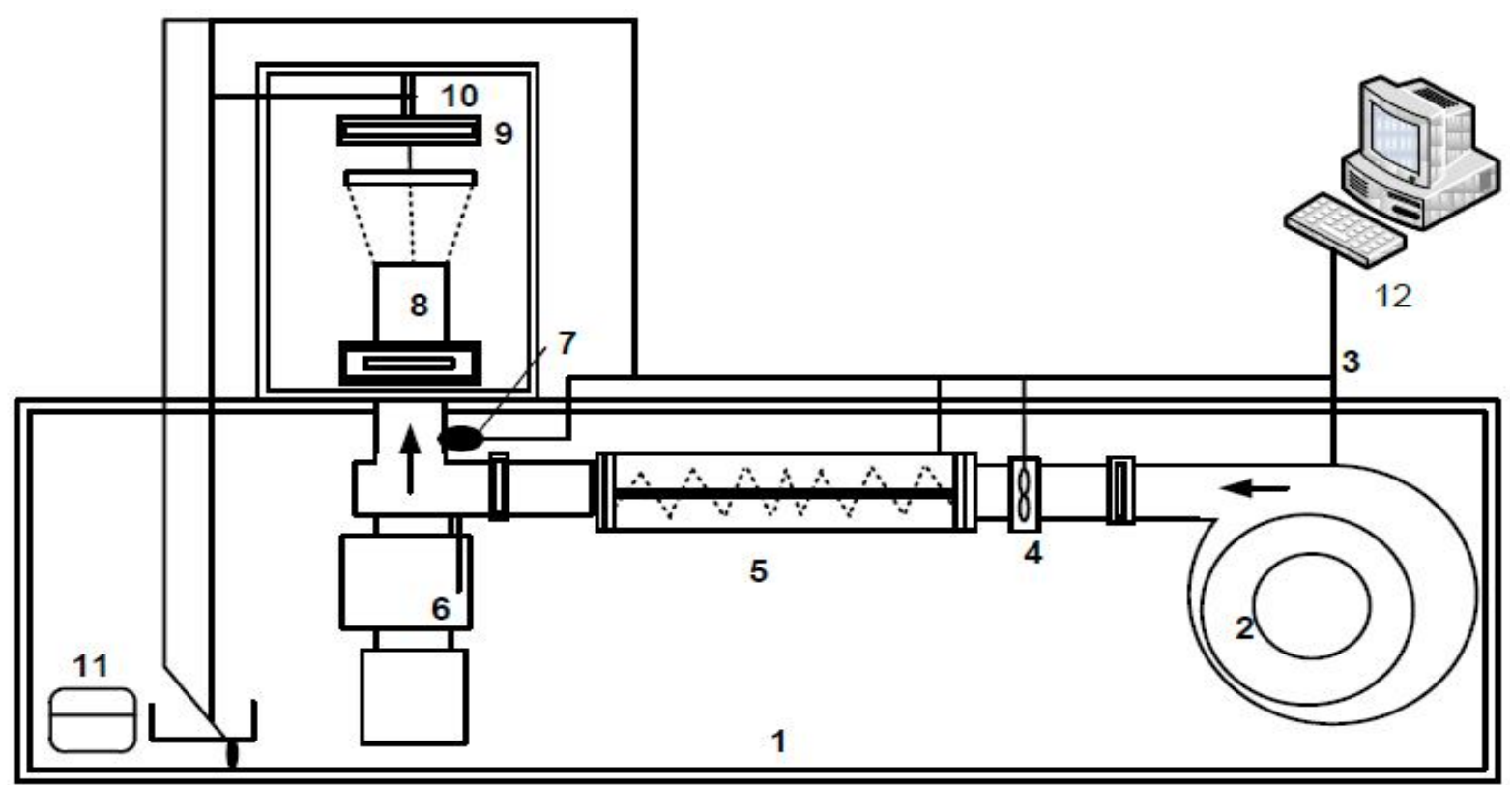

Figura 2. Esquema del secador. 1. Soporte; 2. Ventilador; 3. Control del fluxo del aire; 4. Anemómetro; 5. Resistencias elétricas; 6. Válvula pneumática; 7. Sensor de temperatura; 8. Camara de secado; 9. Balanza; 10. Elevador; 11. Compresor; 12. Ordenador de adquisición de dados e control 
cuando el producto llegaba a un contenido de agua de $10 \%$ b.h.

En ausencia de un método rápido para determinar el contenido de agua de plantas medicinales, el momento de finalizar el proceso de secado fue calculado matemáticamente, obteniéndose el peso final deseado a través de la Ec. 1

$$
\mathrm{m}_{\mathrm{f}}=\mathrm{m}_{\mathrm{i}} \times\left(\frac{100-\mathrm{X}_{\mathrm{i}}}{100-\mathrm{X}_{\mathrm{f}}}\right)
$$

donde:

$\mathrm{m}_{\mathrm{f}}$ - masa final, $\mathrm{g}$

$m_{1}$ - masa inicial, $g$

$X_{i}$ - cantidad inicial de agua, \%.b.h

$\mathrm{X}_{\mathrm{f}}$ - cantidad final de agua, $\% \mathrm{~b} . \mathrm{h}$

Para la realización de este trabajo se realizaron ensayos a diferentes temperaturas de secado $\left(30,40,50,60,70^{\circ} \mathrm{C}\right)$. El diseño experimental fue de bloques completamente aleatorizados, con tres repeticiones. El contenido de agua de equilibrio del tomillo fue determinado por la ecuación de Halsey modificado, propuesto por Soysal \& Oztekin (2001).

$$
X_{e}=\frac{\ln (U R)}{\exp \left(a+b \cdot T^{d}\right)^{-1 / c}}
$$

donde:

$\mathrm{T}$ - temperatura del aire, $\mathrm{K}$

UR - Humedad relativa del aire de secado, $\%$

$\mathrm{a}, \mathrm{b}, \mathrm{c}, \mathrm{d}$ - constantes que dependen de la naturaleza del producto. Para el tomillo:

$$
a=2,97977 ; b=-0,00258 ; c=1,44139 ; d=1,37743
$$

La razón del contenido de agua (RX), durante las diferentes condiciones del aire de secado, fue calculada mediante la Ec. 3

$$
\mathrm{RX}=\frac{\mathrm{W}_{\mathrm{t}}-\mathrm{W}_{\mathrm{e}}}{\mathrm{W}_{0}-\mathrm{W}_{\mathrm{e}}}
$$

donde:

$\mathrm{RX}$ - razón de humedad, adimensional

$\mathrm{W}_{\mathrm{t}}$ - contenido de agua del producto en el tiempo $t$, b.s

$\mathrm{W}_{0}$ - contenido de agua inicial del producto, b.s

Fueron ajustados a 12 diferentes modelos matemáticos citados en la literatura los valores experimentales de RX, para cada temperatura diferente del aire de secado, conforme en la Tabla 1.

La difusividad efectiva fue calculada por la basada en la ley de Fick (Ec. 4), utilizándose ocho termos da serie. La expresión de la razón del contenido de agua asume condiciones de contorno del primero tipo.

$$
\mathrm{RX}=\frac{\mathrm{W}_{\mathrm{t}}-\mathrm{W}_{\mathrm{e}}}{\mathrm{W}_{0}-\mathrm{W}_{\mathrm{e}}}=\frac{8}{\pi^{2}} \cdot \sum_{\mathrm{i}=0}^{\infty} \frac{1}{(2 \mathrm{i}+1)^{2}} \exp \left[-(2 \mathrm{i}+1)^{2} \cdot \pi^{2} \cdot \mathrm{D}_{\mathrm{ef}} \cdot \frac{\mathrm{t}}{4 \mathrm{~L}^{2}}\right]
$$

donde:

$\mathrm{L}$ - media espesura del producto $(\mathrm{m})$. Para el tomillo $\mathrm{L}=$ $0,000851 \mathrm{~m}$

La energía de activación, que expresa la dependencia de la difusividad en función de la temperatura, fue calculada por el logaritmo de la ecuación de Arrhenius.

$$
\ln \left(D_{\text {ef }}\right)=\ln \left(D_{0}\right)-\frac{E_{a}}{R \cdot T}
$$

donde:

$\mathrm{D}_{\mathrm{ef}}$ - difusividad efectiva, $\mathrm{m}^{2} \mathrm{~s}^{-1}$

$\mathrm{D}_{0}$ - factor de Arrhenius, $\mathrm{m}^{2} \mathrm{~s}$

$\mathrm{E}_{\mathrm{a}}$ - energia de activación, $\mathrm{J} \mathrm{mol}^{-1}$

$\mathrm{R}^{\mathrm{a}}$ - constante universal de los gases, 8, $3143 \mathrm{~J} \mathrm{~mol}^{-1} \mathrm{~K}^{-1}$

$\mathrm{T}$ - temperatura, $\mathrm{K}$

Para el ajuste de los modelos matemáticos a los datos experimentales fue realizado el análisis de regresión no lineal, por el método Simplex-Quasi-Newton, para eso utilizamos el

\begin{tabular}{|c|c|c|}
\hline Modelo & Ecuación & Referencia \\
\hline Exponencial simples, 2 parámetros & $R X=a \exp (-k t)$ & Henderson \& Pabis (1961) \\
\hline Exponencial simples, 3 parámetros & $R X=a \exp (-k t)+b$ & Akpinar \& Bicer (2005) \\
\hline Exponencial duplo, 2 parámetros & $R X=a \exp (-k t)+(1-a) \exp (-k a t)$ & Kassem (1998) \\
\hline Exponencial duplo, 3 parámetros & $R X=a \exp (-k t)+(1-a) \exp (-k b t)$ & Sharaf-Elden et al. (1980) \\
\hline Exponencial duplo, 4 parámetros & $R X=a \exp \left(-k_{0} t\right)+b \exp \left(-k_{1} t\right)$ & Henderson (1974) \\
\hline Exponencial triplo, 6 parámetros & $R X=a \exp (-k t)+b \exp \left(-k_{0} t\right)+c \exp \left(-k_{1} t\right)$ & Karathanos (1999) \\
\hline Lewis & $R X=\exp (-k t)$ & Lewis (1921) \\
\hline Page & $R X=\exp \left(-k t^{n}\right)$ & Page (1949) \\
\hline Page modificado & $R X=\exp (-k t)^{n}$ & Overhults et al. (1973) \\
\hline Midilli et al. (2002) & $R X=a \exp \left(-k \cdot t^{n}\right)+b \cdot t$ & Midilli et al. (2002) \\
\hline Thompson & $\left.R X=\exp \left(-a-\left(a^{2}+4 b t\right)^{1 / 2}\right) / 2 b\right)$ & Thompson et al. (1968) \\
\hline Wang \& Singh & $R X=1+a t+b t^{2}$ & Wang \& Singh (1978) \\
\hline
\end{tabular}
programa Statistica $8.0^{\circledR}$, los valores de los parámetros de los modelos fueron estimados en función de la temperatura del aire de secado.

Tabla 1. Modelos matemáticos que fueron utilizados para describir el proceso de secado de las hojas de Thymus vulgaris 
La elección del modelo fue realizada en función del coeficiente de determinación ajustado $\left(\mathrm{R}^{2}\right)$, del error medio relativo (EMR) y del error medio estimado(EME). El error medio relativo y el error medio estimado para cada modelo, fueron determinados por las Ecs. 6 y 7, respectivamente:

$$
\begin{aligned}
& \mathrm{EMR}=\frac{100}{\mathrm{n}} \sum \frac{\left|\mathrm{Y}-\mathrm{Y}_{0}\right|}{\mathrm{Y}} \\
& \mathrm{EME}=\sqrt{\frac{\sum\left(\mathrm{Y}-\mathrm{Y}_{0}\right)^{2}}{\mathrm{GLM}}}
\end{aligned}
$$

donde:

n - número de observaciones

Y - valor observado

$\mathrm{Y}_{0}$ - valor estimado por el modelo

GLM - Número de observaciones menos uno.

\section{RESULTADOS Y DISCUSIÓN}

La humedad relativa del aire de secado fue 50, 32, 17, 9 e $8 \%$ y los valores del contenido de agua en equilibrio obtenidos por medio del modelo de Halsey modificado (Ec. 2) fueran: 8,$56 ; 5,07 ; 3,08 ; 2,03 ;$ e $1,56 \%$ b.h, respectivamente, para el secado con aire a $30,40,50,60$, e $70^{\circ} \mathrm{C}$.

Los valores calculados de la difusividad efectiva para las hojas de tomillo están representados en la Tabla 2. Observase que la difusividad efectiva aumenta con el aumento de la temperatura del aire de secado en el rango de temperaturas con las que hemos trabajado $\left(30\right.$ a $\left.70^{\circ} \mathrm{C}\right)$, evidenciando la reducción de las resistencias internas de difusión de agua con la elevación de la temperatura.

Tabla 2. Valores de la difusividad efectiva $\left(D_{\text {ef }}\right)$ obtenidos para las hojas de tomillo en diferentes temperaturas de secado

\begin{tabular}{cc}
\hline Temperatura $\left(^{\circ} \mathbf{C}\right)$ & Difusividad $\left(\mathbf{m}^{2} \mathbf{s}^{-1}\right)$ \\
30 & $3,689 \times 10^{-12}$ \\
40 & $7,760 \times 10^{-12}$ \\
50 & $2,089 \times 10^{-11}$ \\
60 & $5,626 \times 10^{-11}$ \\
70 & $1,190 \times 10^{-10}$ \\
\hline
\end{tabular}

Los valores de la difusividad efectiva obtenidos en ese trabajo difieren de aquellos de Doymaz (2010) al estudiar el secado de esa misma especie, en el rango de temperatura de 40 a $60{ }^{\circ} \mathrm{C}$ y velocidad del aire de $2 \mathrm{~m} \mathrm{~s}^{-1}$. Ese autor observó que los valores fueron de 1.097 a $5.991 \times 10^{-9} \mathrm{~m}^{2} \mathrm{~s}^{-1}$. Simal et al. (2000) evaluó el secado de las hojas de sábila y el valor de la difusividad efectiva varió desde 5,64 a $18,1 \times 10^{-10} \mathrm{~m}^{2} \mathrm{~s}^{-1}$, para el rango de temperatura de 30 a $70{ }^{\circ} \mathrm{C}$. Panchariya et al. (2002) observaron en el secado de té negro, que la difusividad fue de 1,141 a $2,985 \times 10^{-11} \mathrm{~m}^{2} \mathrm{~s}^{-1}$ para el rango de temperatura de $80 \mathrm{a}$ $120{ }^{\circ} \mathrm{C}$. Therdthai \& Zhou (2009) encontraron para el secado de las hojas de menta, valores de 0,96 e $1,9 \times 10^{-11} \mathrm{~m}^{2} \mathrm{~s}^{-1}$ para la temperatura de 60 e $70^{\circ} \mathrm{C}$, respectivamente.

En la Figura 3, están representados los valores de la difusividad efectiva $\left(D_{\text {ef }}\right)$ en función del revés de la temperatura absoluta del aire de secado.

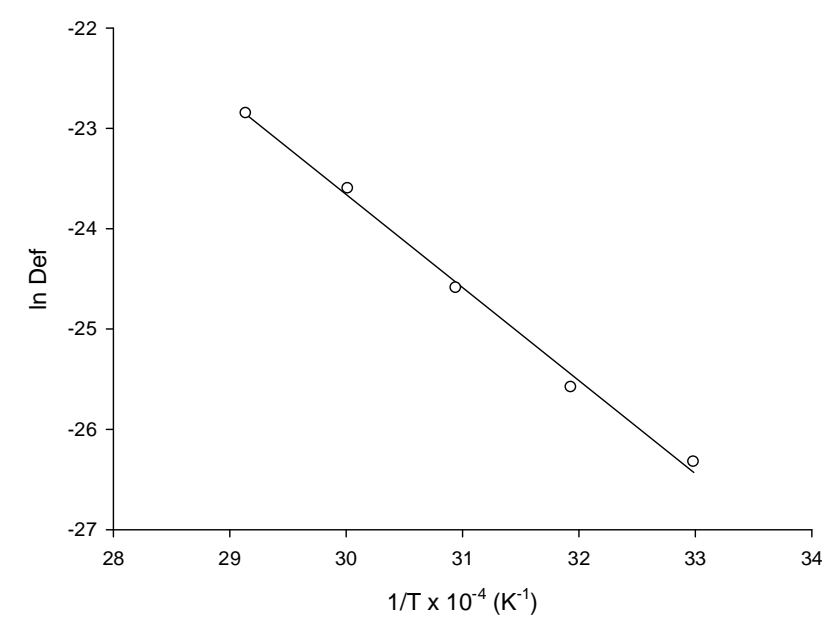

Figura 3. Relación de tipo Arrhenius entre el coeficiente de la difusividad efectiva y la temperatura absoluta del aire de secado de las hojas de tomillo

La energía de activación para la difusión de agua para las hojas de tomillo en condiciones estudiadas fue de $77,16 \mathrm{~kJ} \mathrm{~mol}^{-1}$. Este valor es muy próximo al encontrado por Doymaz (2010) al trabajar en el secado de esta misma especie, siendo el valor encontrado de 73,84 $\mathrm{kJ} \mathrm{mol}^{-1}$. La energía de activación para el secado de diversos productos viene siendo foco de estudios de varios trabajos, como por ejemplo, Simal et al. (2000) donde encontró el valor de la energía de activación de $24,40 \mathrm{~kJ} \mathrm{~mol}^{-1}$ para babosa , 82,93 $\mathrm{kJ} \mathrm{mol}^{-1}$ para menta (Park et al., 2002) y 31,79 $\mathrm{kJ} \mathrm{mol}^{-1}$ para básmamo de limón (Barbosa et al., 2007).

En la Tabla 3 son presentados los valores de los coeficientes de determinación, los errores medios relativos y los errores medios estimados de los modelos matemáticos ajustados a los datos experimentales del secado de las hojas de tomillo. Los coeficientes de determinación por encima de $98 \%$ representan un buen ajuste del modelo matemático para describir el fenómeno de secado (Madamba et al., 1996). De acuerdo con Aguerre et al. (1989), los resultados de errores medios relativos inferiores al $10 \%$ indican un buen ajuste de los valores observados en relación a los ajustados por el modelo en cuestión. El valor del error medio estimado es inversamente proporcional a la capacidad del modelo en describir con fidelidad el proceso de secado (Panchariya et al., 2002).

Observando esa tabla; los resultados de los coeficientes de determinación ajustados $\left(\mathrm{R}^{2}\right)$, los errores medios relativos (EMR) y de los errores medios estimados (EME) indican que el modelo Page y Page modificado son los que mejor describen el proceso de secado de tomillo, independientemente de la temperatura del aire de secado. Por lo tanto, el modelo de Page fue el que mejor se ajusta a los datos experimentales entre los 12 modelos, ya que presentó los mayores valores de coeficiente de determinación $(99,67$ a 99,99\%), menores valores de errores medios relativos $(1,29$ a $9,92 \%)$ y menores errores medios estimados $(0,003$ a 0,019$)$. 
Tabla 3. Valores de los coeficientes de determinación $\left(R^{2}\right)$, errores medios relativos (EMR) y de los errores medios estimados (EME), obtenidos para los modelos matemáticos ajustados a los dados experimentales de secado de las hojas de tomillo

\begin{tabular}{|c|c|c|c|c|c|c|c|c|c|}
\hline $\begin{array}{c}\text { Modelo } \\
\text { matemático }\end{array}$ & $\begin{array}{c}\mathrm{T} \\
\left({ }^{\circ} \mathrm{C}\right)\end{array}$ & $\begin{array}{c}\text { EMR } \\
(\%)\end{array}$ & EME & $\begin{array}{l}R^{2} \\
(\%)\end{array}$ & $\begin{array}{c}\text { Modelo } \\
\text { matemático }\end{array}$ & $\begin{array}{c}\mathrm{T} \\
\left({ }^{\circ} \mathrm{C}\right)\end{array}$ & $\begin{array}{c}\text { EMR } \\
(\%)\end{array}$ & EME & $\begin{array}{l}R^{2} \\
(\%)\end{array}$ \\
\hline \multirow{5}{*}{ Midilli et al. (2002) } & 30 & 5,53 & 0,0151 & 99,754 & \multirow{5}{*}{$\begin{array}{l}\text { Exp. tripo, } 6 \\
\text { parâmetros }\end{array}$} & 30 & 2,71 & 0,0171 & 99,685 \\
\hline & 40 & 59,73 & 0,0840 & 92,817 & & 40 & 9,01 & 0,0187 & 99,658 \\
\hline & 50 & 8,32 & 0,0359 & 98,983 & & 50 & 2,65 & 0,0113 & 99,900 \\
\hline & 60 & 0,78 & 0,0039 & 99,991 & & 60 & 7,14 & 0,0219 & 99,744 \\
\hline & 70 & 11,18 & 0,0115 & 99,910 & & 70 & 60,28 & 0,0533 & 98,897 \\
\hline \multirow{5}{*}{ Page } & 30 & 2,21 & 0,0119 & 99,847 & \multirow{5}{*}{$\begin{array}{l}\text { Exp. duplo, } 3 \\
\text { parâmetros }\end{array}$} & 30 & 4,88 & 0,0300 & 99,017 \\
\hline & 40 & 9,42 & 0,0193 & 99,672 & & 40 & 10,61 & 0,0089 & 99,922 \\
\hline & 50 & 1,29 & 0,0033 & 99,991 & & 50 & 1,53 & 0,0065 & 99,966 \\
\hline & 60 & 250 & 0.0077 & 99.964 & & 60 & 9.19 & 0.0268 & 99.594 \\
\hline & 70 & 9,92 & 0,0141 & 99,949 & & 70 & 19,73 & 0,0184 & 99,861 \\
\hline \multirow{5}{*}{ Lewis } & 30 & 9,67 & 0,0408 & 98,159 & \multirow{5}{*}{ Thompson } & 30 & 5,61 & 0,0192 & 99,597 \\
\hline & 40 & 13,62 & 0,0424 & 98,195 & & 40 & 48,69 & 0,0449 & 97,980 \\
\hline & 50 & 5,46 & 0,0180 & 99,730 & & 50 & 2,53 & 0,0073 & 99,956 \\
\hline & 60 & 9,20 & 0.0254 & 99,594 & & 60 & 21,13 & 0.0557 & 97.736 \\
\hline & 70 & 72,11 & 0,0569 & 98,487 & & 70 & 81,47 & 0,0645 & 98,160 \\
\hline \multirow{5}{*}{$\begin{array}{l}\text { Exp. duplo, } 2 \\
\text { parâmetros }\end{array}$} & 30 & 4,69 & 0,0208 & 99,524 & \multirow{5}{*}{ Wang \& Sing } & 30 & 12,52 & 0,0517 & 97,039 \\
\hline & 40 & 19,00 & 0,0210 & 99,560 & & 40 & 50,16 & 0,7972 & 93,491 \\
\hline & 50 & 0,07 & 0,0048 & 99,981 & & 50 & 13,58 & 0,0376 & 99,884 \\
\hline & 60 & 11,52 & 0,0354 & 99,251 & & 60 & 7,24 & 0,0190 & 99,785 \\
\hline & 70 & 75,16 & 0,0617 & 98,320 & & 70 & 10,19 & 0,0194 & 99,835 \\
\hline \multirow{5}{*}{$\begin{array}{c}\text { Exp. duplo, } 4 \\
\text { parâmetros }\end{array}$} & 30 & 2,47 & 0,0169 & 99,687 & \multirow{5}{*}{ Page modificado } & 30 & 2,33 & 0,0148 & 99,762 \\
\hline & 40 & 8,53 & 0,0201 & 99,602 & & 40 & 9,43 & 0,0194 & 99,672 \\
\hline & 50 & 2,52 & 0,0111 & 99,900 & & 50 & 1,29 & 0,0033 & 99,991 \\
\hline & 60 & 7,18 & 0,0213 & 99,744 & & 60 & 2,50 & 0,0077 & 99,974 \\
\hline & 70 & 60,28 & 0,0516 & 98,897 & & 70 & 9,93 & 0,0143 & 99,959 \\
\hline \multirow{5}{*}{$\begin{array}{l}\text { Exp. simples, } 2 \\
\text { parâmetros }\end{array}$} & 30 & 2,69 & 0,0169 & 99,686 & \multirow{5}{*}{$\begin{array}{l}\text { Exp. simples, } 3 \\
\text { parâmetros }\end{array}$} & 30 & 2,80 & 0,0152 & 99,749 \\
\hline & 40 & 8,98 & 0,0186 & 99,658 & & 40 & 13,13 & 0,0192 & 99,637 \\
\hline & 50 & 2,66 & 0,0110 & 99,900 & & 50 & 3,62 & 0,0095 & 99,928 \\
\hline & 60 & 7,17 & 0,0207 & 99,744 & & 60 & 1,27 & 0,0055 & 99,983 \\
\hline & 70 & 60,28 & 0,0501 & 98,897 & & 70 & 33,90 & 0,0284 & 99,666 \\
\hline
\end{tabular}

En la Tabla 4 son presentados los valores de los parámetros para el modelo de Page, para el secado de las hojas de tomillo en diferentes temperaturas.

Tabla 4. Valores estimados de los parámetros para el modelo de Page para el secado de las hojas de tomillo a diferentes temperaturas de aire

\begin{tabular}{ccc}
\hline $\begin{array}{c}\text { Temperatura } \\
\left({ }^{\circ} \mathbf{C}\right)\end{array}$ & $\mathbf{K}$ & Coeficientes \\
30 & 0,007425 & $\mathbf{N}$ \\
40 & 0,009278 & 0,741418 \\
50 & 0,010665 & 0,784596 \\
60 & 0,012407 & 0,897544 \\
70 & 0,015156 & 1,157269 \\
& & 1,399052 \\
\hline
\end{tabular}

Las ecuaciones para estimar los parámetros " $\mathrm{k}$ " y " $\mathrm{n}$ " del modelo de Page en función de la temperatura del aire de secado son representadas a continuación.

$$
\begin{gathered}
\mathrm{k}=0,0052+0,0000325 * \mathrm{~T}-0,0000015 * \mathrm{~T}^{2} \quad \mathrm{R}^{2}=0,95 \\
\mathrm{n}=0,1520+0,0169 * \mathrm{~T} \quad \mathrm{R}^{2}=0,96
\end{gathered}
$$

Podemos observar en la Tabla 4 que los valores de "k" $y$ " $n$ " aumentan en función del incremento de la temperatura del aire de secado. El parámetro "n" depende de la naturaleza del producto y de las condiciones de secado, evidenciando la resistencia interna del producto al proceso de secado (Misra \& Brooker, 1980).

En la Figura 4, son representadas las curvas de secado elaboradas con los valores observados y estimados por la ecuación de Page, para las hojas de tomillo.

El secado de tomillo (Thymus vulgaris) fue realizada por Doymaz (2010) utilizando temperatura del aire de secado en el rango de 40 a $60^{\circ} \mathrm{C}$, velocidad del aire de $2 \mathrm{~m} \mathrm{~s}^{-1}$ y la humedad relativa de 14 a $45 \%$. Fueron utilizados varios modelos matemáticos a los datos experimentales y el modelo de Midilli et al. (2002) fue que mejor se ajusta a los datos experimentales.

Para el secado de las hojas louro (Laurus nobilis L.), Demir et al. (2004) utilizaron diferentes métodos de secado (aire a 40, 50 y $60{ }^{\circ} \mathrm{C}$, secado a sol y sombra). Entre los diversos modelos matemáticos ajustados a los datos experimentales, el modelo de Page fue el que mejor describía el proceso de secado para las condiciones estudiadas. En estudio realizado con hojas de alecrim (Rosmarinus officinalisL), Arslan \& Ozcan (2008) utilizaron 3 métodos de secado (a sol con temperaturas en un rango de 20 a $30^{\circ} \mathrm{C}$, estufa a $50^{\circ} \mathrm{C}$ y en microondas a $700 \mathrm{~W}$ ). Los autores verificaran que el modelo exponencial simples de 3 parámetros y el de Midilli y Kucuk fueron los más adecuados para describir el proceso de secado realizado mediante el sol y en la estufa, mientras que los modelos de Page, Page 


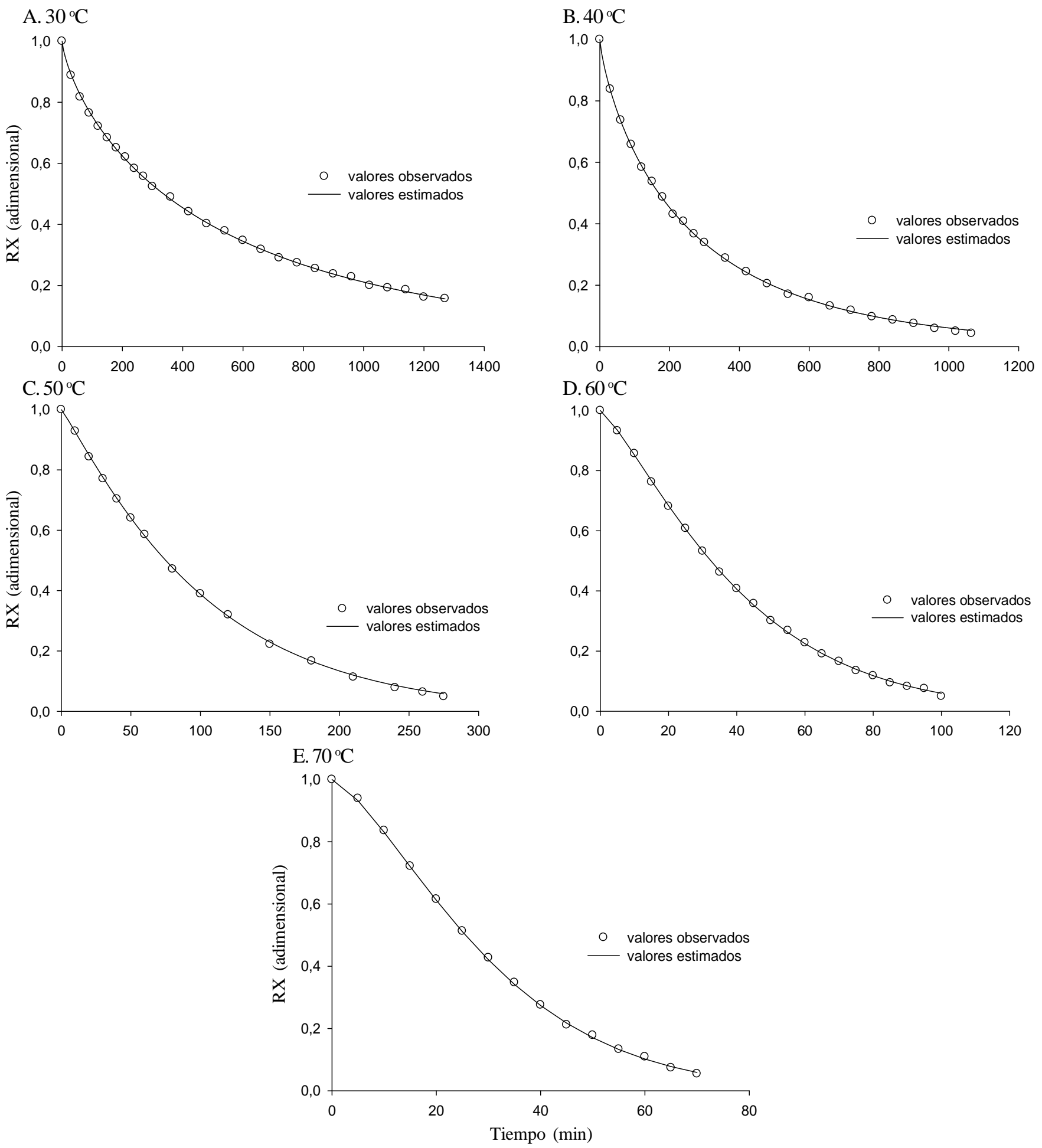

Figura 4. Curvas de secado de tomillo a 30, 40, 50, 60 y $70{ }^{\circ} \mathrm{C}$ ajustadas por el modelo de Page

Modificado, Midilli y Kucuk fueron los que mejor se ajustaban a los datos experimentales de secado con el microondas.

Radünz et al. (2011) utilizó seis temperaturas de aire de secado $\left(40,50,60,70,80\right.$ e $\left.90^{\circ} \mathrm{C}\right)$, con el objetivo de estudiar la cinética de secado de carqueja (Baccharis trimera). Los autores ajustaban los datos experimentales a doce modelos matemáticos, concluyendo que el modelo de Midilli et al. (2002) presentó el mejor ajuste a los datos para el rango de temperatura estudiado ( 40 a $90^{\circ} \mathrm{C}$ ), y el modelo de Page presentó un ajuste adecuado a los datos experimentales para el rango de temperatura de 60 a $90^{\circ} \mathrm{C}$.

\section{Conclusiones}

1. El modelo de Page fue el que mejor se ajustó a los datos experimentales para describir la cinética de secado de las hojas de tomillo. 
2. Los valores de los coeficientes de difusividad efectiva variaron entre $3,689 \times 10^{-12} \mathrm{e} 1,190 \times 10^{-10} \mathrm{~m}^{2} \mathrm{~s}^{-1}$.

3. El valor de energía de activación para la difusión del agua en el secado de tomillo fue de $77,16 \mathrm{~kJ} \mathrm{~mol}^{-1}$.

\section{Agradecimentos}

Al CNPq y FAPEMIG por el apoyo financiero para la realización del trabajo.

\section{LITERATURA CITADA}

Aguerre, R. J.; Suarez, C.; Viollaz, P. E. New BET type multilayer sorption isotherms - Part II: Modelling water sorption in foods. Lebensmittel-Wissenschaft and Technologie, v.22, p.192-195, 1989.

Akpinar, E. K.; Bicer, Y. Modelling of the drying of eggplants in thin-layers. International Journal of Food Science and Technology, v.40, p.1-9, 2005.

Andrade, E. T.; Borém, F. M.; Hardoim, P. R. Cinética de secagem do café cereja, bóia e cereja desmucilado, em quatro diferentes tipos de terreiros. Revista Brasileira de Armazenamento, v.1, p.37-43, 2003.

AOAC - Association of Official Analytical Chemists: Official methods of analysis. $16^{\mathrm{a}}$ ed. W. Horwitz: Washington, 1997. 850p.

Arslan, D.; Ozcan, M. M. Evaluation of drying methods with respect to drying kinetics, mineral content and colour characteristics of rosemary leaves. Energy Conversion and Management, v.49, p.1258-1264, 2008.

Arslan, D.; Ozcan, M. M.; Menges, H. O. Evaluation of drying methods with respect to drying parameters, some nutritional and colour characteristics of peppermint (Mentha x piperita L.). Energy Conversion and Management, v.51, p.2769-2775, 2010.

Bagamboula, C. F.; Uyttendaele, M.; Debevere, J. Inhibitory effect of thyme and basil essential oils, carvacrol, thymol, estragol, linalool and p-cymene towards Shigella sonnei and S. flexneri. Food Microbiology, v.21, p.33-42, 2004.

Barbosa, F. F.; Melo, E. C.; Santos, R. H. S.; Rocha, R. P.; Martinazzo, A. P.; Radünz, L. L.; Gracia, L. M. N. Evaluation of mathematical models for prediction of thinlayer drying of brazilian lemon-scented verbena leaves (Lippia alba (Mill) N.E. Brown). Revista Brasileira de Produtos Agroindustriais, v.9, p.71-80, 2007.

Centeno, S.; Calvo, M. A.; Adelantado, C.; Figuero, S. Antifungal activity of extracts of Rosmarinus officinalis and Thymus vulgaris against Aspergillus flavus and A. ochraceus. Pakistan Journal of Biological Sciences, v.13, p.452-455, 2010.

Christensen, C. M.; Kaufmann, H. H. Microflora. In: Christensen, C. M. Storage of cereal grain and their products. St. Paul: American Association of Cereal Chemists, 1974. p.158-192.
Daferera, D. J.; Ziogas, B. N.; Polissiou, M. G. CG/EManalysis of essential oils from some Greek aromatic plants and their fungitoxicity on Penicillium digitatum. Journal Agriculturae Food Chemistry, v.48, p.2576-2581, 2000.

Demir, V.; Gunhan, T.; Yagcioglu A. K.; Degirmencioglu, A. Mathematical modelling and the determination of some quality parameters of air-dried bay leaves. Biosystems Engineering, v.88, p.325-335, 2004.

Doymaz, I. Drying of thyme (Thymus vulgaris L.) and selection of a suitable thin-layer drying model. Journal of Food Processing and Preservation, v.1, p.1-8. 2010.

Henderson, S. M. Progress in developing the thin layer drying equation. Transactions of ASAE. v.17, p.1167-1172, 1974.

Henderson, S. M.; Pabis, S. Grain drying theory I. Temperature effect on drying coefficient. Journal of Agriculture Engineering Research, v.6, p.169-174, 1961.

Karathanos, V. T. Determination of water content of dried fruits by drying kinetics. Journal of Food Engineering, v.39, p.337344, 1999.

Kassem, A. S. Comparative studies on thin layer drying models for wheat. In: International Congress on Agricultural Engineering, 13, 1998, Rabat. Anais... Rabat: ANAFID, v.6, 1998. p.2-6.

Lewinsohn, E.; Dudai, N.; Tadmor, Y.; Katzir, I.; Ravid, U.; Putievsky, E.; Joel, D. M. Histochemical localization of citral accumulation in lemongrass leaves (Cymbopogon citratus (DC.) Stapf, Poaceae). Annals Botany, v.81, p.35-39, 1998.

Lewis, W. K. The rate of drying of solids materials. The Journal of Industrial and Engineering Chemistry, v.13,p.427-432, 1921.

Madamba, P. S.; Driscoll, R. H.; Buckle, K. A. Thin layer drying characteristics of garlic slices. Journal of Food Engineering, v.29, p.75-97, 1996.

Mewes, S.; Kruger, H.; Pank, F. Physiological morphological chemical and genomic diversities of different origins of thyme (Thymus vulgaris L.). Genetic Resources Crop Evolution, v.55, p.1303-1311, 2008.

Midilli, A.; Kucuk, H.; Vapar, Z. A new model for single-layer drying. Drying Technology, v.20, p.1503-1513, 2002.

Misra, M. K.; Brooker, D. B. Thin-layer drying and rewetting equation for shelled yellow corn. Transactions of the ASAE, v.23, p.1254-1260, 1980.

Novacosk, R.; Torres, R. S. A. Atividade antimicrobiana sinérgica entre óleos ssenciais de lavanda (Lavandula officinalis), melaleuca (Melaleuca alternifolia), cedro (Juniperus irginiana), tomilho (Thymus vulgaris) e cravo (Eugenia caryophyllata). Revista Analytica, v.21, p.36-39, 2006.

Overhults, D. D.; White, G. M.; Hamilton, M. E.; Ross, I. J. Drying soybeans with heated air. Transactions of the ASAE, v.16, p.195-200, 1973.

Page, G. E. Factors influencing the maximum rates of air drying shelled corn in thin layers. Indiana: Universidade de Purdue, 1949. Dissertação Mestrado

Panchariya, P. C.; Popovic, D.; Sharma, A. L. Thin-layer modeling of black tea drying process. Journal of Food Engineering, v.52, p.349-357, 2002. 
Park, K. J.; Vohnikova, Z.; Brod, F. P. R. Evaluation of drying parameters and desorption isotherms of garden mint leaves (Mentha crispa L.). Journal of Food Engineering, v.1, p.193199, 2002.

Porte, A.; Godoy, R. L. O. Alecrim (Rosmarinus Officinalis L.): Propriedades antimicrobiana e química do óleo essencial. Boletim do CEPPA, v.19, p.193-210, 2001.

Radünz, L. L.; Amaral, A. S.; Mossi, A. J.; Melo, E. C.; Rocha, R. P. Avaliação da cinética de secagem de carqueja. Engenharia na Agricultura, v.19, p.19-27, 2011.

Reis, M. S.; Mariot, A.; Steenbock, W. Diversidade e domesticação de plantas medicinais. In: Simões, C. M. O. Farmacognosia: Da planta ao medicamento. Porto Alegre/ Florianópolis: UFRGS/ UFSC, 2003. p.3-74.

Sharaf-Elden, Y. I.; Blaisdell, J. L.; Hamdy, M. Y. A model for ear corn drying.Transactions of the ASAE, v.5, p.1261-1265, 1980.

Simal, S.; Femenía, A.; Llull, P.; Rosselló, C. Dehydration of aloe vera: simulation of drying curves and evaluation of functional properties. Journal of Food Enginnering, v.43, p.109-114, 2000.
Sokoviæ, M. D.; Vukojeviæ, J.; Marin, P. D.; Brkiæ, D.; Vajs, V.; Griensven, L. J. L. D. van. Chemical composition of essential oils of thymus and mentha species and their antifungal activities. Molecules, v.14, p.238-249, 2009.

Soysal, Y.; Oztekin, S. Comparison of seven equilibrium moisture content equations for some medicinal and aromatic plants. Journal of Agriculture Engineering Research, v.78, p.57-63, 2001.

Therdthai, N.; Zhou, W. Characterization of microwave vacuum drying and hot air drying of mint leaves (Mentha cordifolia Opiz ex Fresen). Journal of Food Engineering, v.91, p.482489, 2009.

Thompson, T. L.; Peart, R. M.; Foster, G. H. Mathematical simulation of corn drying - A new model. Transactions of ASAE, v.11, p.582-586, 1968.

Venskutonis, P.R. Effect of drying on the volatile constituents of thyme (Thymus vulgaris L.) and sage (Salvia officinalis L.). Food Chemistry, v.59, p.219-227, 1997.

Wang, C. Y.; Singh, R. P. Use of variable equilibrium moisture content in modeling rice drying. Transactions of ASAE, v.78, p.6505, 1978 . 